\title{
Pteridófitas ocorrentes em fragmentos de Floresta Serrana no estado de Pernambuco, Brasil
}

\author{
Sergio Romero da Silva Xavier ${ }^{1}$ \\ Iva Carneiro Leão Barros ${ }^{2}$
}

\begin{abstract}
Resumo
Foi realizado um levantamento florístico e análise de aspectos ecológicos das pteridófitas ocorrentes no brejo de Serra Negra em Bezerros, estado de Pernambuco, Brasil. Caracterizada como Brejo de Altitude ou Floresta Serrana, a área de estudo apresenta flora diferenciada da vegetação semi-árida que a cerca, por isso, os Brejos de Altitude, em especial a Serra Negra de Bezerros, atualmente estão sofrendo acelerado processo de devastação. Em um período de um ano, foram encontradas 31 táxons específicos, sendo a família Polypodiaceae a mais representativa com $46 \%$ dos registros. A espécie Trichomanes angustifrons (Fée) Wess. Boer destaca-se como novo registro para o Nordeste do Brasil. Os aspectos ecológicos que predominaram no brejo de Serra Negra de Bezerros foram o hábito herbáceo, o habitat rupícola, o tipo de ambiente mesófilo, a forma de vida hemicriptófita e os afloramentos rochosos como ambientes preferenciais. $\mathrm{O}$ fator condicionante da área ainda possuir certa diversidade específica, mesmo com a ampla restrição da mata nativa é que foram mantidas importantes condições ambientais necessárias para a sobrevivência da pteridoflora, como temperaturas baixas, umidade relativa do ar elevada e garoa noturna e matinal sempre presente.
\end{abstract}

Palavras-chave: Bezerros, Brejo de Altitude, Floresta Serrana, Pernambuco, pteridófitas.

\begin{abstract}
A floristic survey and analysis of ecological aspects of the pteridophytes occurring at the brejo of Serra Negra, Municipality of Bezerros, State of Pernambuco - Brazil, was conducted. The study site, characterized as Brejo de Altitude or Floresta Serrana, presents a flora that distinguishes itself from the surrounding semi-arid vegetation. Therefore, the Brejos de Altitude and particularly the Serra Negra of Bezerros are currently going through a very intense devastation process. Thirtyone specific taxa have been found in a period of a year, among which the Polypodiaceae is well represented comprising $46 \%$ of these registers. The species Trichomanes angustifrons (Fée) Wess. Boer stands out as a new register for Northeastern Brazil. The predominant ecological aspects observed at the brejo of Serra Negra of Bezerros were herbaceous habit, lithophytic habitat, a mesophylous type of environment, life form hemicryptophyte and the rocky formations as preferential environments. Despite the highly restricted native vegetation, the area still owns conditioning factors for the occurrence of a certain diversity of species. These factors are important environmental conditions such as low temperatures, high relative air humidity, and night and morning mist. These conditions have been maintained, and are important and necessary to the survival of the pteridophyte flora.
\end{abstract}

Keywords: Bezerros, Brejo de Altitude, Floresta Serrana, Pernambuco, pteridophytes.

${ }^{1}$ Programa de Pós-Graduação em Biologia Vegetal da Universidade Federal de Pernambuco, Brasil. xaviersergio@yahoo.com.br

2Departamento de Botânica, Universidade Federal de Pernambuco, Brasil. ivaleao@ truenet.com.br 


\section{INTRODUÇÃO}

A zona fitogeográfica do Agreste do estado de Pernambuco, caracteriza-se por apresentar vegetação semi-árida dominante chamada Caatinga e em alguns pontos isolados, uma outra vegetação bem mais exuberante, mantida pela altitude e por ventos úmidos, conhecida como Floresta Serrana ou Brejo de Altitude e classificada por Andrade-Lima (1966) como Floresta Ombrófila Densa que constitui em Pernambuco, disjunções da Floresta Tropical Perenifólia, dentro da Zona da Caatinga (Andrade-Lima, 1960). De acordo com vários autores, os Brejos de Altitude nordestinos são refúgios florísticos de florestas úmidas que penetraram no interior do continente há milhares de anos e que recuaram com as variações climáticas que ocorreram na época, deixando ilhas de vegetação florestal serrana em meio ao domínio do semi-árido(Coimbra-Filho \& Câmara, 1996; Prance, 1982 e Bigarella et al., 1975).

As pteridófitas, também conhecidas como fetos, samambaias ou avencas, na sua maioria são plantas dependentes de condições de umidade e sombreamento, favorecendo a fixação deste grupo vegetal nas regiões de mata onde se formam estes tipos de microambientes. As pteridófitas são plantas freqüentes nas regiões de mata devido ao microclima formado nestes corpos florestais (Barros \& Silva, 1996). Dessa forma, qualquer desmatamento compromete seriamente a sobrevivência destes verdadeiros "fósseis vivos" que têm o seu ambiente natural destruído. Estas plantas são, portanto, ótimas indicadoras do grau de preservação de uma área florestal. Andrade-Lima (1966, 1970) já alertava para o desmatamento das áreas de Brejo de Altitude em Pernambuco, que possuem um conjunto de condições atrativas para a agricultura, em especial a do café.

Nos Brejos de Altitude do estado de Pernambuco, poucos levantamentos florísticos de pteridófitas foram desenvolvidos, como Santos \& Barros (1999) para as matas de Biturí Grande no município de Brejo da Madre de Deus e Santiago (2002) para três fragmentos no município de Bonito.
A Serra Negra do município de Bezerros é uma área caracterizada como Brejo de Altitude que está sofrendo acelerado processo de devastação. Este trabalho visa contribuir para um maior conhecimento florístico e ecológico das áreas de Brejo de Altitude do estado de Pernambuco, através do estudo da sua flora pteridofítica. Fornece, ainda, subsídios para pesquisas futuras das mais diversas naturezas a respeito desses vegetais, bem como a preservação deste ambiente, totalmente diferenciado do domínio do semi-árido que o cerca, sendo considerado um refúgio biológico de grande valor.

\section{MATERIAIS E MÉTODOS}

\section{Caracterização da Área de estudo}

Na bacia hidrográfica do Rio Ipojuca, encontra-se o município de Bezerros, distante 100 $\mathrm{km}$ de Recife, capital do Estado. No Nordeste da sede do município, numa região que integra a porção oriental do Planalto da Borborema, ergue-se o Brejo de Serra Negra, favorecido por boa exposição aos ventos úmidos do sudoeste (Andrade-Lima, 1966) (Fig. 1). Nesta região, houve uma acentuada ação antrópica para fins agrícolas de forma que a antiga cobertura florestal que antes dominava, hoje se encontra bastante reduzida com poucos indivíduos arbóreos, remanescentes da antiga floresta (Sales et al., 1998), em certos casos, substituída por espécies apropriadas ao sombreamento do café, uma das lavouras típicas da região (Andrade-Lima, 1966).

Dentro do distrito de Serra Negra duas áreas foram escolhidas para a realização das coletas de campo: os Sítios Vertentes e Freixeiras, constituindo 150 ha. Nestas áreas, as altitudes variam entre 800 e $950 \mathrm{~m}$. No Sítio Vertentes encontra-se o último fragmento florestal ainda preservado na Serra Negra, que atualmente encontra-se administrada pela Prefeitura do município. O Sítio Freixeiras, localizado ao leste do Sítio Vertentes, apresenta características vegetacionais que não diferem muito de grande parte das áreas do distrito de Serra Negra de Bezerros, ou seja, presença 


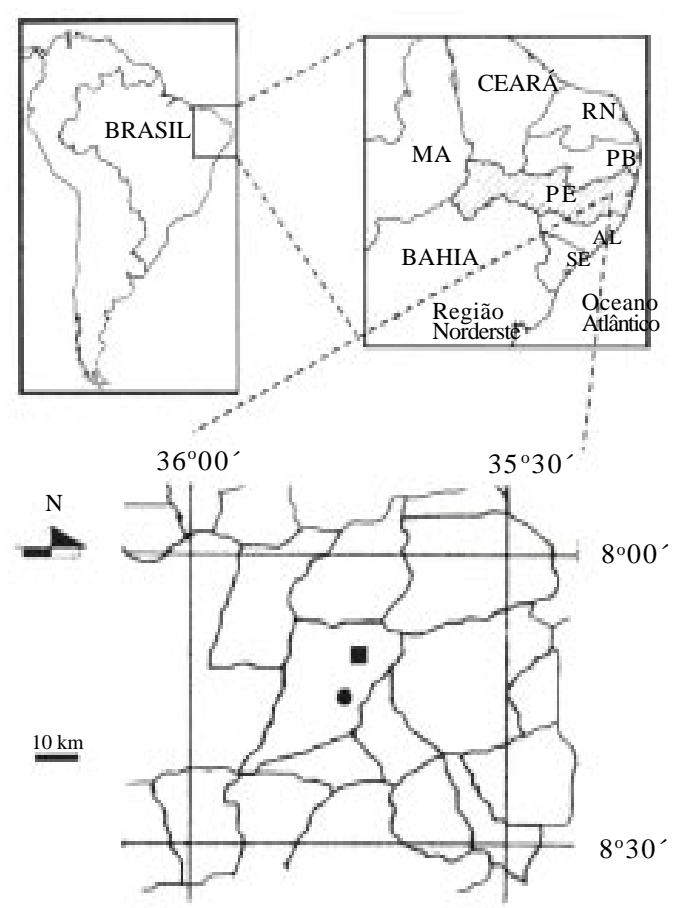

Figura 1 - Mapa de localização do brejo de Serra Negra no município de Bezerros, estado de Pernambuco, Brasil. (- Bezerros; Serra Negra)

de alguns remanescentes arbóreos da floresta destruída, predominância de cafezais e grande número de afloramentos rochosos.

Em grandes clareiras e áreas abertas, observa-se que espécies nativas da vegetação da Caatinga circundante se instalaram nestes ambientes e em alguns pontos já dominam quase completamente, comprometendo, assim, a vegetação nativa. Essa susceptibilidade à semi-aridez da região circunvizinha evidencia a fragilidade do equilíbrio ecológico nos Brejos de Altitude (Sales et al., 1998).

\section{Coleta, análise, identificação e cataloga- ção das espécies}

Entre os meses de dezembro de 1998 e outubro de 1999, foram realizadas sete coletas à Serra Negra de Bezerros. Para a identificação e apresentação das espécies, no texto do trabalho foi adotado o Sistema de Classificação proposto por Kramer \& Green (1990) e utilizadas as Chaves Analíticas contidas nos trabalhos de Sehnem (1967, 1968a, 1968b, 1970, 1971, 1972, 1974, 1979), Stolze (1976, 1981), Tryon \& Tryon (1982), Proctor (1985), Mickel \& Beitel (1988), Tryon \& Stolze (1989a, 1989b, 1991, 1992, 1993), Moran \& Riba (1995) e Kramer \& Green (1990).

Após a identificação, o material foi depositado no Herbário Professor Geraldo Mariz (UFP) da Universidade Federal de Pernambuco.

Para a comparação do estágio sucessional em cada um dos fragmentos, foi realizado um levantamento das espécies não restritas a ambientes florestais e ocorrentes nas áreas de estudo, consultando os trabalhos de Barros (1997), Salino (2000) e Sylvestre (2001).

Também foram feitas observações ecológicas das pteridófitas em seus microhabitats, abordando hábitos, habitats, tipos de ambientes e Formas de Vida segundo Barros (1997) além dos ambientes preferenciais segundo Ambrósio \& Barros (1997) com modificações para este estudo.

\section{RESULTADOS}

No levantamento das pteridófitas da Serra Negra de Bezerros, foram encontradas 10 famílias, 15 gêneros e 31 espécies (Tab. 1). A família melhor representada é Polypodiaceae (14 spp), seguida de Aspleniaceae (6 spp), Thelypteridaceae (3 spp), Hymenophyllaceae (2 spp), Blechnaceae, Schizaeaceae, Pteridaceae, Vittariaceae, Dennstaedtiaceae e Dryopteridaceae (todas com uma espécie). (Fig. 2).

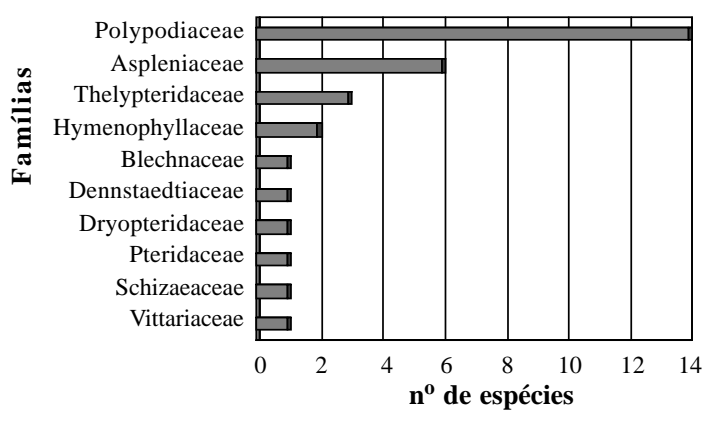

Figura 2 -Representatividade das famílias de pteridófitas ocorrentes no Brejo de Serra Negra no município de Bezerros, estado de Pernambuco, Brasil. 
Tabela 1 - Levantamento das espécies de pteridófitas ocorrentes na Serra Negra de Bezerros nos Sítios Vertentes e Freixeiras. Todas as espécies foram coletadas por Xavier ou Xavier et al. X, Presente

\begin{tabular}{|c|c|c|c|}
\hline Famílias & Espécies & Vertentes & Freixeiras \\
\hline Aspleniaceae & $\begin{array}{l}\text { Asplenium clausseni Hieron. } \\
\text { Asplenium cristatum } \mathrm{Lam} . \\
\text { Asplenium formosum } \mathrm{Willd} \text {. } \\
\text { Asplenium praemorsum } \mathrm{Sw} . \\
\text { Asplenium pumilum } \mathrm{Sw} . \\
\text { Asplenium serratum } \mathrm{L} .\end{array}$ & $\begin{array}{l}\mathbf{X} \\
\mathbf{X} \\
\mathbf{X} \\
\mathbf{X}\end{array}$ & $\mathbf{X}$ \\
\hline Blechnaceae & Blechnum occidentale $\mathrm{L}$. & $\mathbf{X}$ & $\mathbf{X}$ \\
\hline Dennstaedtiaceae & Pteridium aquilinum (L.) Kuhn & $\mathbf{X}$ & $\mathbf{X}$ \\
\hline Dryopteridaceae & Polybotrya cylindrica Kaulf. & $\mathbf{X}$ & \\
\hline Hymenophyllaceae & $\begin{array}{l}\text { Trichomanes angustifrons (Fée) Wess. Boer } \\
\text { Trichomanes ovale (E. Fourn.) Wess. Boer }\end{array}$ & $\begin{array}{l}\mathbf{X} \\
\mathbf{X}\end{array}$ & \\
\hline Polypodiaceae & $\begin{array}{l}\text { Campyloneurum phyllitidis (L.) C. Presl } \\
\text { Campyloneurum repens (Aubl.) C. Presl } \\
\text { Microgramma geminata (Schrad. ) R.M. Tryon \& A.F. Tryon } \\
\text { Microgramma lycopodioides (L.) Copel. } \\
\text { Microgramma squamulosa (Kaulf.) de la Sota } \\
\text { Microgramma vacciniifolia (Langsd. \& Fisch.) Copel. } \\
\text { Niphidium crassifolium (L.) Lellinger } \\
\text { Pecluma ptilodon (Kunze) M. G. Price } \\
\text { Pleopeltis astrolepis (Liebm.) E. Fourn. } \\
\text { Pleopeltis macrocarpa (Willd.) Kaulf. } \\
\text { Polypodium aureum L. } \\
\text { Polypodium dissimile L. } \\
\text { Polypodium hirsutissimum Raddi } \\
\text { Polypodium triserialeSw. }\end{array}$ & $\begin{array}{l}\mathbf{X} \\
\mathbf{X} \\
\mathbf{X} \\
\mathbf{X} \\
\mathbf{X} \\
\mathbf{X} \\
\mathbf{X} \\
\mathbf{X} \\
\mathbf{X} \\
\mathbf{X}\end{array}$ & $\begin{array}{l}\mathbf{X} \\
\mathbf{X} \\
\mathbf{X} \\
\mathbf{X} \\
\mathbf{X} \\
\mathbf{X}\end{array}$ \\
\hline Pteridaceae & Hemionitis tomentosa $($ Lam.) Raddi & & $\mathbf{X}$ \\
\hline Schizaeaceae & Lygodium venustum $\mathrm{Sw}$. & $\mathbf{X}$ & \\
\hline Thelypteridaceae & $\begin{array}{l}\text { Thelypteris hispidula (Decne.) C. F. Reed } \\
\text { Thelypteris interrupta(Willd.) B.C. Stone } \\
\text { Thelypteris serrata (Cav.) Alston }\end{array}$ & $\begin{array}{l}\mathbf{X} \\
\mathbf{X} \\
\mathbf{X}\end{array}$ & \\
\hline Vittariaceae & Ananthacorus angustifolius (Sw.) Underw. \& Maxon & & $\mathbf{X}$ \\
\hline
\end{tabular}

A espécie Trichomanes angustifrons (Fée) Boer destacou-se na Serra Negra de Bezerros como uma nova ocorrência para o Nordeste do Brasil. Foi encontrada no Sítio Vertentes como rupícola de hábito herbáceo em ambiente ciófilo no interior da mata, apresentando ainda, forma de vida hemicriptófita.

Quanto às áreas estudadas no Brejo de Serra Negra de Bezerros, o Sítio Freixeiras destaca-se por apresentar uma maior representatividade de espécies não restritas a ambientes florestais $(86,66 \%)$. Por outro lado, no Sítio Vertentes, o percentual de espécies nestas condições representa 56,52\%.
Os Sítios Freixeiras e Vertentes na Serra Negra de Bezerros, estão em processo de preservação há poucos anos, no entanto, evidenciam a marcante ação antrópica expressa pelo plantio do café e da banana, amplamente praticado anos atrás. Talvez sendo esta a razão de não possuir uma flora pteridofítica ainda mais rica.

Em se tratando dos aspectos ecológicos da pteridoflora estudada, a grande maioria apresentou o hábito herbáceo com exceção de Polybotrya cylindrica e Lygodium venustum que apresentaram hábito arbustivo-escandente e Pteridium aquilinum, sub-arbustivo ereto. 
O habitat rupícola se destacou na área de estudo seguido pelos habitats holocorticícola, terrícola, solos alagáveis e hemicorticícola (Fig. 3).

Em relação ao tipo de ambiente, as espécies mesófilas destacaram-se, seguidas pelas espécies ciófilas, heliófilas e higrófilas (Fig. 4).

Com a exceção de Pteridium aquilinum, que apresentou a forma de vida geófita, Thelypteris interrupta, fanerófita, Polybotrya cylindrica e Campyloneurum repens, ambas hemiepífitas, todas as demais espécies

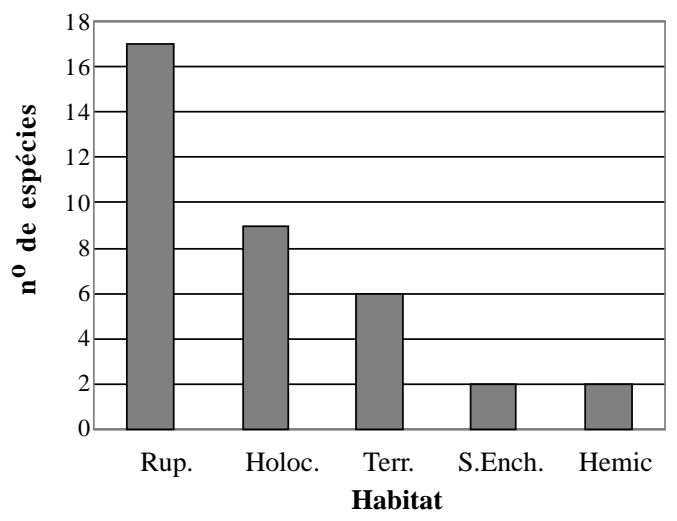

Figura 3 - Tipos de habitats encontrados nas espécies de pteridófitas no Brejo de Serra Negra, município de Bezerros, estado de Pernambuco, Brasil (Rup.= Rupícola; Holoc. $=$ Holocorticícola; Terr. $=$ Terrícola; S. Ench. $=$ Solos Encharcados; Hemic. $=$ Hemicorticícola).

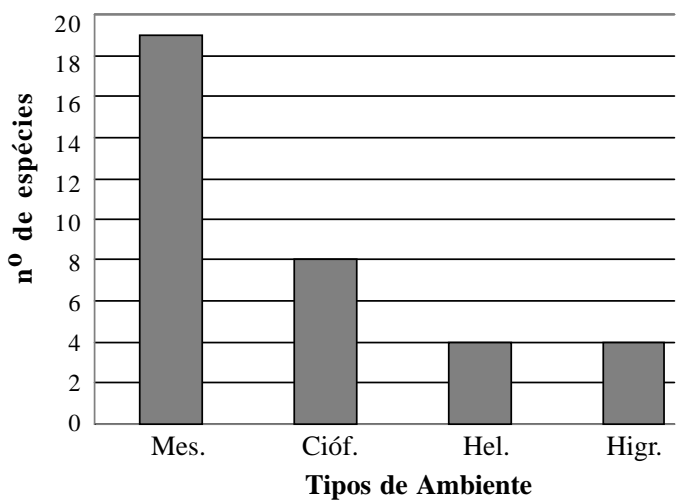

Figura 4 -Tipos de Ambientes de ocorrência das espécies de pteridófitas na Serra Negra do município de Bezerros, estado de Pernambuco, Brasil (Mes.= Mesófilo; Cióf.= Ciófilo; Hel.= Heliófilo; Higr.= Higrófilo). enquadraram-se como hemicriptófitas e epífitas (Fig. 5).

Quanto aos ambientes preferenciais, apenas Thelypteris hispidula e Blechnum occidentale foram registradas junto a córregos. As demais espécies encontraramse, nos afloramentos rochosos, nas margens de trilhas no interior da mata, encostas, clareiras, paredões rochosos e margens da mata (Fig. 6).

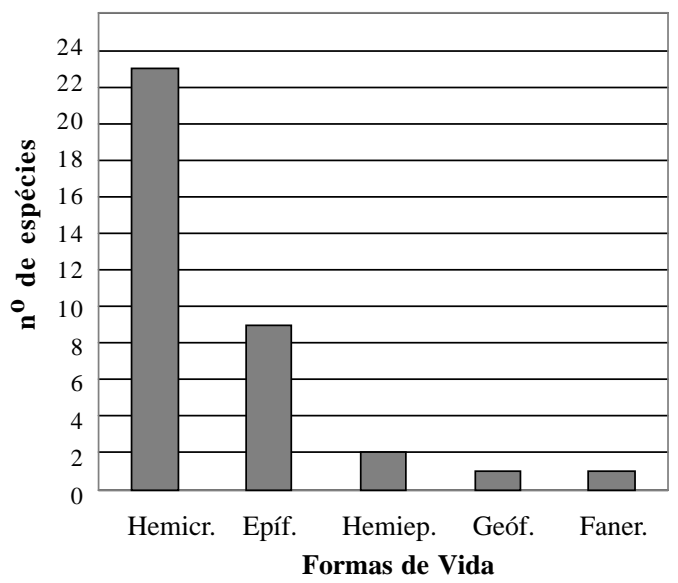

Figura 5 - Formas de Vida apresentadas pelas pteridófitas ocorrentes no brejo de Serra Negra no município de Bezerros, estado de Pernambuco, Brasil $($ Hemicr. $=$ Hemicriptófita; Epíf. $=$ Epífita; Hemiep.= Hemiepífita; Geóf.= Geófita; Faner.= Fanerófita).

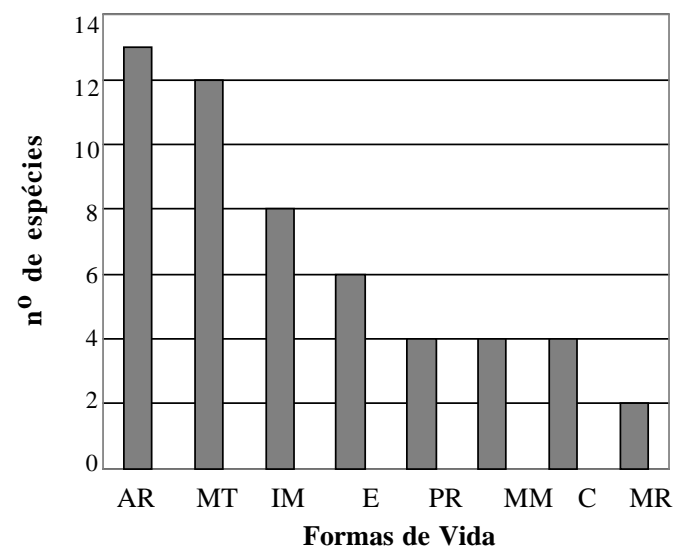

Figura 6 - Ambientes Preferenciais das pteridófitas ocorrentes no Brejo de Serra Negra, município de Bezerros, estado de Pernambuco, Brasil (AR = Afloramentos rochosos; $\mathrm{MT}=$ Margens de trilhas; $\mathrm{IM}=$ Interior da Mata; $\mathrm{E}=$ Encostas; $\mathrm{PR}=$ Paredões rochosos; $\mathrm{MM}=$ Margens da Mata; $\mathrm{C}=$ Clareiras; $\mathrm{MR}=$ Margens de regatos). 
Observou-se que, apesar da ampla restrição da mata nativa, o Brejo de Serra Negra de Bezerros ainda possui certa diversidade de pteridófitas, que se concentram em afloramentos rochosos localizados em meio aos cafezais, sombreados por remanescentes arbóreos da antiga floresta. Foram mantidas na área importantes condições climáticas ambientais necessárias para a sobrevivência da pteridoflora, como temperaturas baixas, umidade relativa do ar elevada e garoa noturna e matinal sempre presente.

\section{DISCUSSÃO}

Os Brejos de Altitude são pequenas ilhas de matas sobre maciços isolados; são à rigor, disjunções das matas, enquadrando-se como áreas de florestas úmidas a sub-úmidas, em topos de serras ou próximas aos relevos tabulares residuais (Andrade-Lima, 1970).

Condicionadas pelo relevo, as florestas tropicais serranas, alcançam algumas serras da caatinga de Pernambuco, com altitudes em torno de $1.000 \mathrm{~m}$, o que propicia uma diminuição de temperatura e um aumento das precipitações locais (Andrade-Lima, 1961). Sendo este o comportamento verificado na Serra Negra de Bezerros.

Como as pteridófitas acompanham estas condições de disponibilidade hídrica (Brade, 1940), ocorrem com maior ou menor número de espécies, segundo estes fatores.

A significativa representatividade de uma família caracteristicamente epífita e de gêneros também evidentemente epífitos, refletem o comentário de Sota (1971), onde afirma que essas plantas alcançam os ambientes dos troncos e ramos das copas das árvores em busca de uma condição de vida melhor; onde ocorrem temperaturas mais baixas e umidade mais elevada, por vezes até, em detrimento da falta de alimentos.

Comparando os dados obtidos na área estudada, com os de Paula (1993), para a Serra do Baturité no Ceará, a pteridoflora do Baturité apresenta-se bem mais rica, com o registro de 92 espécies; no entanto, é bem mais ampla e melhor estudada; apesar destes fatos, apresenta concordância quanto à ocorrência das espécies Hemionitis tomentosa, Asplenium formosum, A. pumilum, Microgramma lycopodioides, Pleopeltis macrocarpa, P. astrolepis e Polypodium aureum, espécies de ocorrência comum nas Florestas Serranas do Nordeste brasileiro.

Em analogia com os dados referidos por Barros et al. (1988), com respeito às pteridófitas registradas exclusivamente nos Florestas Serranas de Pernambuco, há completa concordância na ocorrência das espécies Hemionitis tomentosa, Polypodium hirsutissimum, Niphidium crassifolium e Pleopeltis astrolepis, presentes na Serra Negra e que representam $60 \%$ entre os registros exclusivos para este tipo de ecossistema no Estado.

É importante comentar também, que a espécie Asplenium pumilum, anteriormente coletada em Pernambuco, apenas na Serra dos Ventos, no município de Belo Jardim, também Brejo de Altitude, foi registrada para o Sítio Freixeiras, na Serra Negra de Bezerros, ocorrendo em ricas populações e muito bem representada.

Dentre as 65 espécies citadas por Barros et al. (1988), como ocorrentes nos Brejos de Altitude estudados no estado de Pernambuco, 14\% foram coletadas nos Sítios Freixeiras e Vertentes na Serra Negra de Bezerros neste presente estudo. O Sítio Vertentes é um pouco mais rico em ocorrência de espécies, pelo próprio estado de preservação de sua vegetação.

Comparando os Sítios Vertentes e Freixeiras, este último apresenta um maior número de espécies não restritas a ambientes florestais, o que pode sugerir que esta localidade encontra-se em um estágio sucessional menos avançado. Confirmando esta relação, Lima et al. (1997) afirmam que as espécies generalistas habitam preferencialmente os bordos de mata e áreas degradadas.

Os dados obtidos neste trabalho são 
muito significativos, pois trazem uma importante contribuição ao conhecimento da biodiversidade das pteridófitas em remanescentes de Floresta Serrana no estado de Pernambuco. Destacase ainda, o fato de que estes ambientes, altamente fragmentos e que provavelmente abrigavam floras outrora mais ricas, oferecem condições bem diferenciadas do macroclima regional segundo relato do CPRH (1994), sugerindo assim, direcionamentos para a conservação desses ecossistemas.

\section{AGRADECIMENTOS}

Os autores agradecem ao Conselho Nacional de Desenvolvimento Científico e Tecnológico $(\mathrm{CNPq})$ pela concessão de bolsa de estudo ao primeiro autor e à Universidade Federal de Pernambuco, por fornecer transporte para deslocamento até a área de pesquisa, além das instalações locais e aos colegas colaboradores Conceição de Paula, Marcio Pietrobom e Washington Vasconcelos.

\section{REFERÊNCIAS BIBLIOGRÁFICAS}

Ambrósio, S. \& Barros, I.C.L. 1997. Pteridófitas de uma área remanescente de Floresta Atlântica do Estado de Pernambuco, Brasil. Acta Botânica Brasilica. 11(2): 105-113.

Andrade-Lima, D. de. 1960. Estudos fitogeográficos de Pernambuco. Arquivos do Instituto de Pesquisas Agronômicas. 5: 305-341.

Andrade-Lima, D. de. 1961. Tipos de Florestas de Pernambuco. Anais da Associação de Geógrafos Brasileiros. 12: 69-85.

Andrade-Lima, D. de. 1966. Esboço fitoecológico de alguns brejos de Pernambuco. Boletim Técnico do Instituto de Pesquisas Agronômicas de Pernambuco. 8: 1-27.

Andrade-Lima, D. de. 1970. Recursos Vegetais de Pernambuco. Boletim Técnico do Instituto de Pesquisas Agronômicas de Pernambuco. 41: 1-32.

Barros, I.C.L. 1997. Pteridófitas ocorrentes em Pernambuco: ensaio biogeográfico e análise numérica. Recife, Tese de Doutorado, Universidade Federal Rural de Pernambuco, $577 \mathrm{p}$.

Barros, I.C.L. \& Silva, M.B.C. 1996. Taxonomia, Padrão de Venação e Aparelhos Estomáticos de Pteris schwackeana Chris. (Pteridaceae/Pteridophyta). Boletim da Sociedade Broteriana. 67(2): 257-262.

Barros, I.C.L.; Silva, A.R.J. \& Lira, O.C. 1988. Distribuição geográfica das pteridófitas ocorrentes no estado de Pernambuco. Acta Botanica Brasilica. 2(1-2): 47-84.

Bigarella, J.J.; Andrade-Lima, D. de \& Riehs, P.J. 1975. Considerações a respeito das Mudanças Paleoambientais na Distribuição de algumas espécies vegetais e animais do Brasil. Anais da Academia Brasileira de Ciências, 47 (suplemento).

Brade, A.C. 1940. Contribuição para o estudo da Flora Pteridofítica da Serra do Baturité, estado de Ceará. Rodriguésia 4(13): 289314 ,

Coimbra-Filho, A. \& Câmara, I.G. 1996. Os limites originais do bioma Mata Atlântica na Região Nordeste do Brasil. Rio de Janeiro, Fundação Brasileira para a Conservação da Natureza (FBCN), 82 p.

CPRH - Companhia Pernambucana de Recursos Hídricos 1994. Diagnóstico para recuperação do Parque Ecológico João Vasconcelos Sobrinho. Companhia Pernambucana de Recursos Hídricos, Recife.

Kramer, K.U. Green, P.S. 1990. Pteridophytes and Gymnosperms. In: Kubitzki, K. The Families and Genera of Vascular Plants. New York, Springer-Verlag, 1: 1-404.

Lima, H.C. de; Lima, M.P.M. de; Vaz, A.M.S. da F.; Pessoa, S. de V.A. 1997. Trepadeiras da Reserva Ecológica de Macaé de Cima. In: Lima, H.C. de \& Guedes-Bruni, R.R. Serra de Macaé de Cima: Diversidade, Florística e Conservação em Mata Atlântica. Rio de Janeiro, Jardim Botânico do Rio de Janeiro, p. 75-87. 
Mickel, J.T. \& Beitel, M.J. 1988. Pteridophyte Flora of Oaxaca. New York, The New York Botanical Garden, 568p.

Moran, R.C. \& Riba, R. 1995. Flora Mesoamericana. Psilotaceae a Salviniaceae. México, Universidad Nacional Autônomo de México. 1: 1-470.

Paula, E.L. 1993. Pteridófitas da Serra do Baturité-Ceará. Recife, Dissertação de Mestrado, Universidade Federal de Pernambuco, $196 \mathrm{p}$.

Prance, G.T.A. 1982. A review of the phytogeographic evidences for Pleidtocene climate change in the Neotropics. Annals of Missouri Botanical Garden, 69: 594-624.

Proctor, G.R. 1985. Ferns of Jamaica: A guide to Pteridophytes. London, British Museum Natural History, 631p.

Sales, M.F.; Mayo, S.J. \& Rodal, M.J.N. 1998. Plantas vasculares das Florestas Serranas de Pernambuco: Um Checklist da Flora Ameaçada dos Brejos de Altitude, Pernambuco, Brasil. Recife, Imprensa Universitária Universidade Federal Rural de Pernambuco, $130 \mathrm{p}$.

Salino, A. 2000. Estudos taxonômicos na família Thelypteridaceae (Polypodiopsida) no estado de São Paulo, Brasil, Campinas, Tese de Doutorado, Universidade Estadual de Campinas, $327 \mathrm{p}$.

Santiago, A.C.P. 2002. Pteridófitas ocorrentes em três fragmentos florestais de um Brejo de Altitude (Bonito-Pernambuco-Brasil). Recife Dissertação de Mestrado, Universidade Federal Rural de Pernambuco, 80 p.

Santos, K.M.R. \& Barros, I.C.L. 1999. Pteridófitas das Matas do Bituri Grande, Município de Brejo da Madre de Deus, estado de Pernambuco, Brasil. Memórias da Sociedade Broteriana. 40 (1): 1-140.
Sehnem, A. 1967. Vitariáceas. In: Reitz, R. Flora Ilustrada Catarinense. Itajaí, Herbário Barbosa Rodrigues, 18p.

Sehnem, A. 1968a. Aspleniáceas. In: Reitz, R. Flora Ilustrada Catarinense. Itajaí, Herbário Barbosa Rodrigues, 96p.

Sehnem, A. 1968b. Blecnáceas. In: Reitz, R. Flora Ilustrada Catarinense. Itajaí, Herbário Barbosa Rodrigues, 90p.

Sehnem, A. 1970. Polipodiáceas. In: Reitz, R. Flora Ilustrada Catarinense. Itajaí, Herbário Barbosa Rodrigues, 173p.

Sehnem, A. 1971. Himenofiláceas. In: Reitz, R. Flora Ilustrada Catarinense. Itajaí, Herbário Barbosa Rodrigues, 98p.

Sehnem, A. 1972. Pteridáceas. In: Reitz, R. Flora Ilustrada Catarinense. Itajaí, Herbário Barbosa Rodrigues, 244p.

Sehnem, A. 1974. Esquizeáceas. In: Reitz, R. Flora Ilustrada Catarinense. Itajaí, Herbário Barbosa Rodrigues, 78p.

Sehnem, A. 1979. Aspidiáceas. In: Reitz, R. Flora Ilustrada Catarinense. Itajaí, Herbário Barbosa Rodrigues, 360p.

Sota, E.R. de La. 1971. El epifitismo y las pteridofitas en Costa Rica (América Central). Nova Hedwigia. 21: 401-465.

Stolze, R.G. 1976. Ferns and fern allied of Guatemala. Ophioglossaceae. Through Cyatheaceae. Part I. Fieldiana Museum Natural History. 39: 1-130. Chicago.

Stolze, R.G. 1981. Ferns and fern allies of Guatemala. Part II. Polypodiaceae. Fieldiana 6: $1-522$.

Sylvestre, L.S. 2001. Revisão taxonômica das espécies de Aspleniaceae A.B. Frank ocorrentes no Brasil, São Paulo, Tese de Doutorado, Universidade de São Paulo, 571 p.

Tryon, R.M. \& Stolze, R.G. 1989a. Pteridophyta of Peru. Part I. 1. Ophioglossaceae- 12. Cyatheaceae. Fieldiana. 27: 1-145. 1989b. Pteridophyta of Peru. Part II. 
13. Pteridaceae - 15. Dennstaedtiaceae.

Fieldiana. 22: 1-128.

1991. Pteridophyta of Peru. Part IV.

17. Dryopteridaceae. Fieldiana. 27: 1176.

1992. Pteridophyta of Peru. Part III. 16. Thelypteridaceae. Fieldiana 29: 180.

1993. Pteridophyta of Peru. Part V. 18. Aspleniaceae-21. Polypodiaceae. Fieldiana. 32: 1-190.

Tryon, R.M. \& Tryon, A.F. 1982. Ferns and Allieds plants with Special References to Tropical America. New York, Springer-Verlag, 867p. 